\title{
The Neural Mechanism of Acupuncture Analgesia
}

\author{
Qiu-ling XU ${ }^{1,2, a}$ and Tao $\operatorname{LIU}^{1,3, b,{ }^{*}}$ \\ ${ }^{1}$ Hainan Medical University, Haikou 571101, China \\ ${ }^{2}$ Doctor, professor. Research direction: Neurobiological mechanism of acupuncture. \\ ${ }^{3}$ Professor, Research direction: Neurobiological mechanism of acupuncture. \\ axq17809@163.com \\ b13647549720@163.com \\ "Corresponding author
}

Keywords: Acupuncture analgesia, Neural Mechanism.

\begin{abstract}
The acupuncture signal in the uplink conduction, one side through the spinal cord segmental relationship affect the adjacent segments dominated by the skin, visceral activity and adjacent segments of the pain in the introduction; more often up to reach the brain stem, Forebrain and other parts, through the activation of high central release of impulse to achieve the analgesic effect. So we study the neural mechanism of acupuncture analgesia from Spinal cord level integration, the brain stem and thalamus level of integration and the integration of the limbic system.
\end{abstract}

\section{Introduction}

Chronic pain is a serious threat to human health disease, not only bring patients body and mental suffering, to reduce the quality of life, at the same time increase the economic burden of family and society. Acupuncture as a safe analgesia method, not only has better clinical curative effect, and has better performance. Then we study the neural mechanism of acupuncture analgesia.

\section{Spinal Cord Level Integration}

Acupuncture-induced analgesia depends on the activation of endogenous pain modulation pathways[1]. Tobaldini $G$ asked whether ascending nociceptive control (ANC), a form of pain-induced analgesia, contributes to the antinociceptive effect of acupuncture. To answer this question, they tested the ability of procedures that block ANC-induced analgesia, at peripheral, spinal, nucleus accumbens and rostral ventral medulla levels, to block acupuncture-induced analgesia.

Weng ZJ[2] study that investigate the role of the purinergic receptor P2X3 in the peripheral and central nervous systems during acupuncture treatment for the visceral pain of irritable bowel syndrome (IBS). Electroacupuncture (EA) could downregulate the expression of the $\mathrm{P} 2 \mathrm{X} 3$ receptor and ease the sensitivity to visceral pain. The $\mathrm{P} 2 \mathrm{X} 3$ receptor plays an important role in IBS visceral pain. The different levels of $\mathrm{P} 2 \mathrm{X} 3$ in the peripheral enteric nervous system and central nervous system mediate the effects of the EA treatment of the visceral hyperalgesia of IBS. Inhibitory Effects of Scolopendra Pharmacopuncture on the Development and Maintenance of Neuropathic Pain in Rats: Possible Involvement of Spinal Glial Cells.[3] Scolopendra extracts were used for pharmacopuncture at the Kidney 1 acupoint to investigate the role of 
Scolopendra pharmacopuncture (SPP) in both the development and maintenance of neuropathic pain induced by L5 spinal nerve ligation in rats and the contribution of spinal glial cells. A single treatment and five once-daily treatments with SPP were given to evaluate its effects on the development and maintenance stages of neuropathic pain, respectively, which was followed by behavioral tests. Immunohistochemistry and Western blotting tests were also carried out. A single treatment of SPP delayed spinal nerve ligation-induced mechanical allodynia and thermal hyperalgesia and induced a profound decrease in the expression of ionized calcium binding adaptor protein in the lumbar spinal cord. Repeated SPP treatments reliably suppressed mechanical allodynia and thermal hyperalgesia at later time points, and these results correlated mainly with decreases in glial fibrillary acidic protein. Intriguingly, ionized calcium binding adaptor protein expression was also reduced after repeated SPP. These results illustrate that neuropathic pain in the development and maintenance stages is alleviated by SPP treatment, which may be ascribed principally to deactivations of microglia and astroglia, respectively. Additionally, microglial inactivation seems to be partially involved in preventing neuropathic pain in the maintenance stage.Hsu SF[4] objected that spinal p38 activity and analgesic effect after low- and high-intensity electroacupuncture stimulation in a plantar incision rat model. They demonstrated that 10-mA EA exerts a significant inhibition against post-PI mechanical hypersensitivity via a p38-independent pathway. Importantly, co-treatment with low-dose p38 inhibitor and 3-mA EA can counteract spinal phospho-p38 to exert strong analgesic effect. Our finding suggests a novel strategy to improve EA analgesic quality. Liang Y[5]found electroacupuncture attenuates spinal nerve ligation-induced microglial activation mediated by p38 mitogen-activated protein kinase. Xu J[6] found electroacupuncture Relieves Nerve Injury-Induced Pain Hypersensitivity via the Inhibition of Spinal P2X7 Receptor-Positive Microglia. Jiang QY [7] found that electroacupuncture relieves labour pain and influences the spinal dynorphin/kappa-opioid receptor system in rats.

\section{The Brain Stem and Thalamus Level of Integration}

Recently, a series of studies have given rise to and provided evidence for the hypothesis that the nucleus submedius $(\mathrm{Sm})$ in the medial thalamus is involved in modulation of nociception. The Sm, ventrolateral orbital cortex (VLO) and the periaqueductal gray (PAG) constitute a pain modulatory pathway, activation of which leads to activation of the PAG-brainstem descending inhibitory system and depression of the nociceptive inputs in the spinal cord and trigeminal nucleus. Other studies have indicated that the Sm-VLO-PAG pathway plays an important role in the analgesia induced by electroacupuncture stimulation of the acupuncture point (acupoint) for exciting small diameter fiber (A-delta and C group) afferents. Opioid peptides, serotonin, dopamine, glutamate and their related receptors are involved in Sm- and/or VLO-mediated descending antinociception, and a GABAergic disinhibitory mechanism participates in mediating the antinociception induced by activation of mu-opioid receptors, serotonin 1 (A) receptors, and dopamine $\mathrm{D}(2)$-like receptors. This review describes these findings, which provide important new insights into the roles of the thalamus and cerebral cortex in descending pain modulation[8]. Advanced neuroimaging approaches have been employed to prove that migraine was a central nervous system disorder. This study aims to examine resting-state abnormalities in 
migraine without aura (MWoA) patients stratified by disease duration, and to explore the neuroimaging markers for reflecting the disease duration. [9] Compared with healthy controls, MWoA patients with long-term disease duration showed comprehensive neuronal dysfunction than patients with short-term disease duration. In addition, increased average ReHo values in the thalamus, brain stem, and temporal pole showed significantly positive correlations with the disease duration. On the contrary, ReHo values were negatively correlated with the duration of disease in the anterior cingulate cortex, insula, posterior cingulate cortex and superior occipital gyrus. Yeo S [10]found neuroprotective changes of thalamic degeneration-related gene expression by acupuncture in an MPTP mouse model of parkinsonism: microarray analysis. Functional neuroimaging studies suggest[11] that a lateral network in the brain is associated with the sensory aspects of pain perception while a medial network is associated with affective aspects. The highest concentration of opioid receptors is in the medial network. There is significant evidence that endogenous opioids are central to the experience of pain and analgesia. We applied an integrative multimodal imaging approach during acupuncture. We found functional magnetic resonance imaging signal changes in the orbitofrontal cortex, insula, and pons and diprenorphine positron emission tomography signal changes in the orbitofrontal cortex, medial prefrontal cortex, insula, thalamus, and anterior cingulate cortex. These findings include brain regions within both the lateral and medial pain networks.

\section{The Integration of the Limbic System}

Acupuncture-related modulation of pain-associated brain networks during electrical pain stimulation: a functional magnetic resonance imaging study[12]. Findings of existing functional MRI (fMRI) studies on the neural mechanisms that mediate effects of acupuncture analgesia are inconsistent. This study analyzes the effects of manual acupuncture on pain ratings and brain activation in response to experimental, electrical pain stimuli. Fourteen healthy volunteers were examined by using a 1.5-T MRI scanner. The intensity of pain stimuli was adjusted to individual pain ratings on a numeric rating scale. Baseline fMRI was performed during electrical pain stimulation in a blocked design. For the second session, manual acupuncture with repeated stimulation was performed on contralateral acupoints-large intestine 4 , liver 3 , and stomach 36-before imaging. After imaging, subjective pain ratings and ratings of the de qi sensation were assessed. Compared with baseline, volunteers showed modulated brain activity under pain conditions in the cingulate gyrus, insula, primary somatosensory cortex, and prefrontal areas after the acupuncture session. In accordance with the literature, anterior insular and prefrontal activity seemed to be correlated with acupuncture treatment. This study supports the existence of analgesic acupuncture effects that outlast the needling period. Pain-associated brain areas were modulated in direct response to a preceding acupuncture treatment. Gao $\mathrm{YH}$ found [13] activation of hippocampal MEK1 contributes to the cumulative antinociceptive effect of electroacupuncture in neuropathic pain rats. EA intervention can induce time-dependent cumulative analgesia in neuropathic pain rats after 4 successive sessions of daily EA intervention, which is at least in part related to the activation of hippocampal MEK1. Lee IS[14] studied to compare behavioral and functional brain responses to the act of inserting needles into the body in two different contexts, 
treatment and stimulation, and to determine whether the behavioral and functional brain responses to a subsequent pain stimulus were also context dependent. Twenty-four participants were randomly divided into two groups: an acupuncture treatment (AT) group and an acupuncture stimulation (AS) group. Each participant received three different types of stimuli, consisting of tactile, acupuncture, and pain stimuli, and was given behavioral assessments during fMRI scanning. Although the applied stimuli were physically identical in both groups, the verbal instructions differed: participants in the AS group were primed to consider the acupuncture as a painful stimulus, whereas the participants in the AT group were told that the acupuncture was part of therapeutic treatment. Acupuncture yielded greater brain activation in reward-related brain areas (ventral striatum) of the brain in the AT group when compared to the AS group. Brain activation in response to pain stimuli was significantly attenuated in the bilateral secondary somatosensory cortex and the right dorsolateral prefrontal cortex after prior acupuncture needle stimulation in the AT group but not in the AS group. Inserting needles into the body in the context of treatment activated reward circuitries in the brain and modulated pain responses in the pain matrix. Our findings suggest that pain induced by therapeutic tools in the context of a treatment is modulated differently in the brain, demonstrating the power of context in medical practice.

\section{Conclusion}

Acupuncture analgesia has been recognized at home and abroad and widely used. Acupuncture analgesic mechanism need further study, for the clinical treatment of acupuncture to provide a theoretical basis.

\section{Acknowledgement}

This research supported by: National Natural Science Foundation of China (No.81360560, No.81660820).

\section{References}

[1] Tobaldini G, Aisengart B, Lima MM, Tambeli CH, Fischer L. J Pain. 2014. 15(4): 422-34.

[2] Weng ZJ, Wu LY, Zhou CL, et al. Purinergic Signal. 2015. 11(3): 321-9.

[3] Li C, Ji BU, Lee JE, et al. J Acupunct Meridian Stud. 2015. 8(5): 236-44.

[4] Hsu SF, Zeng YJ, Tsai SY, et al. Life Sci. 2015. 128: 15-23.

[5] Liang Y, Du JY, Qiu YJ, Fang JF, Liu J, Fang JQ. Chin J Integr Med. 2016. 22(9): 704-13.

[6] Xu J, Chen XM, Zheng BJ, Wang XR. Anesth Analg. 2016. 122(3): 882-92.

[7] Jiang QY, Wang MY, Li L, et al. Acupunct Med. 2016. 34(3): 223-8.

[8] Tang JS, Qu CL, Huo FQ. Prog Neurobiol. 2009. 89(4): 383-9.

[9] Zhao L, Liu J, Dong X, et al. J Headache Pain. 2013. 14: 85.

[10] Yeo S, Choi YG, Hong YM, Lim S. Gene. 2013. 515(2): 329-38. 
[11] Dougherty DD, Kong J, Webb M, Bonab AA, Fischman AJ, Gollub RL. Behav Brain Res. 2008. 193(1): 63-8.

[12] Theysohn N, Choi KE, Gizewski ER, et al. J Altern Complement Med. 2014. 20(12): 893-900.

[13] Gao YH, Li CW, Wang JY, et al. BMC Complement Altern Med. 2016. 16(1):517.

[14] Lee IS, Wallraven C, Kong J, et al. Physiol Behav. 2015. 140: 148-55. 